\title{
A LINEAR UPPER BOUND IN ZERO-SUM RAMSEY THEORY
}

\author{
YAIR CARO \\ Department of Mathematics \\ School of Education \\ University of Haifa - ORANIM \\ Tivon 36-910, Israel
}

(Received May 19, 1992)

\begin{abstract}
Let $n, r$ and $k$ be positive integers such that $k \mid\left(\begin{array}{l}n \\ r\end{array}\right)$. There exists a constant $c(k, r)$ such that for fixed $k$ and $r$ and for every group $A$ of order $k$

$$
R\left(K_{n}^{r}, A\right) \leq n+c(k, r)
$$

where $R\left(K_{n}^{r}, A\right)$ is the zero-sum Ramsey number introduced by Bialostocki and Dierker [1], and $K_{n}^{r}$ is the complete $r$-uniform hypergraph on $n$-vertices.
\end{abstract}

KEY WORDS AND PHRASES. Ramsey numbers, zero-sum, complete hypergraph. 1991 AMS SUBJECT CLASSIFICATION CODE. 05 C55.

\section{INTRODUCTION.}

In 1961, Erdös, Ginzburg and Ziv proved the following theorem.

THEOREM A. [2] Let $m \geq k \geq 2$ be positive integers such that $k \mid m$. Let $\left\{a_{1}, a_{2}, \cdots, a_{m+k-1}\right\}$ be a collection of integers. There exists a subset $I \subset\{1,2, \cdots, m+k-1\}$, $|I|=m$ such that $\sum_{i \in I} a_{i} \equiv 0(\operatorname{modk})$.

This theorem was the starting point of the seminal paper of Bialostocki and Dierker [1] in which they introduced the concept of zero-sum subsets, which we generalize below.

In 1976, Olson proved a deep generalization of the Erdös-Ginzburg-Ziv theorem, namely:

THEOREM B. [3]. Let $m \geq k \geq 2$ be positive integers such that $k \mid m$. Let $A$ be a group of order $k$ and let $B=\left\{b_{1}, \cdots, b_{m+k+1}\right\}$ be a sequence of elements of $A$. Then there is a set of $m$ indices $I=\left\{i_{1}, i_{2}, \cdots, i_{m}\right\}$ such that $\sum_{j=1}^{m} b_{i},=0($ in $A)$.

Observe however that we cannot conclude that $i_{1}<i_{2}<\cdots<i_{m}$, hence we possibly rearrange a subsequence of length $m$ to obtain the desired presentation of the unit of $A$.

Let $K_{n}^{r}$ denote the complete $r$-uniform hypergraph on $n$ vertices. Let $c: E\left(K_{n}^{r}\right) \rightarrow A$ be a mapping of all the edges ( $r$-subsets) of $K_{n}^{r}$ into the group $A$ of order $k$. Such a coloring will be called an $A$-coloring. A subhypergraph $H \subset K_{n}^{r}$ is called zero-sum $(\bmod A)($ with respect to $c)$ if for some ordering of the edges of $H \sum_{e \in E(H)} c(e)=0$, where 0 is the unit of $A$ with the binary operation written additively.

Let $H$ be an $r$-uniform hypergraph and define the zero-sum Ramsey number of $H$ with respect to a group $A$ as follows:

$R(H, A)$ is the minimal integer $n$ such that for every $A$-coloring of the edges of $K_{n}^{r}$ there is a zero-sum $(\bmod A)$ copy of $H$ in $K_{n}^{r}$. 
We shall use the notation $e(H)$ for the number of edges of a hypergraph $H$. The existence of $R(H, A)$ in the case that $|A| \mid \epsilon(H)$ follows from the existence of the classical Ramsey number $R(H, k)$ (using $k$ colors), because if $k \mid e(H)$ then of course $R(H, A) \leq R(H, k)$, regardless of the structure of the group $A$. Hence we assume from now on that $|A| \mid e(H)$.

The determination of the order of magnitude of $R\left(K_{n}, Z_{k}\right), k \mid\left(\begin{array}{l}n \\ 2\end{array}\right)$ has been considered in [4] and [5]. (See also the many articles given in the references). In [4], it is shown that $R\left(K_{n}, Z_{k}\right) \leq n+c(k)$ (for $k \mid\left(\begin{array}{l}n \\ 2\end{array}\right)$ and $c(k)=R\left(K_{3 k-1}, k\right)$.

In [5], $c(k)$ has been reduced, using algebraic tools, to $2 k-2$ for $k$ being a prime power and $n$ large, and to $k(k+1)(k+2) \log _{2} k$ for arbitrary $k$ and $n$ large. The method used in [5] is not suitable to deal with the determination of $R\left(K_{n}^{r}, A\right)$ when $A$ is not a cyclic group, hence our technique will be based on that of [4].

We shall not make any effort to obtain the best possible constant $c(k, r)$ mentioned in the abstract, rather proving its existence. We emphasize here that, unlike the classical Ramsey numbers, the zero-sum Ramsey numbers are not monotone in the sense that $H \subset G$ does not imply $R(H, A) \leq R(G, A)$ (even if $|A||e(H)| A|| e,(G)$ ) and many examples of the nonmonotone property are known (see e.g., [6], [5]).

Our proof relies only on Ramsey's theorem and can be thought of as a combinatorial generalization of Olson's theorem.

\section{THE MAIN RESULT.}

THEOREM. Let $n, r$ and $k$ be positive integers such that $k \mid\left(\begin{array}{l}n \\ r\end{array}\right)$ and let $A$ be a group of order $k$. There exists a constant $c(k, r)$ such that $R\left(K_{n}^{r}, A\right) \leq n+c(k, r)$. We need first two simple lemmas.

LEMMA 1. Let $r, k \geq 2$ be positive integers. Then for every $1 \leq i \leq r, k \mid\left(\begin{array}{c}r ! k \\ i\end{array}\right)$.

PROOF. Trivial by direct checking of the binomial coefficient

$$
\left(\begin{array}{c}
r ! k \\
i
\end{array}\right)=\frac{(r ! k)(r ! k-1) \cdots(r ! k-i+1)}{i !} \equiv 0(\bmod k) \text { because } i ! \mid r !
$$

LEMMA 2. Let $r, k \geq 2$ be positive integers. Then for every integer $n, k \mid\left(\begin{array}{l}n \\ r\end{array}\right)$ iff $k \mid\left(\begin{array}{c}n+r ! k \\ r\end{array}\right)$. PROOF. Write

$$
(\underset{r}{n+r ! k})=\frac{[n+r ! k][(n-1)+r ! k][(n-2)+r ! k] \cdots[(n-r+1)+r ! k]}{r !}=\left(\begin{array}{l}
n \\
r
\end{array}\right)+k N
$$

for some integer $N$, and the lemma follows.

PROOF OF THE THEOREM. Write $n=m+(t-1) r ! k, 1 \leq m \leq r ! k$ and $k \mid\left(\begin{array}{l}n \\ r\end{array}\right)$. We shall prove by induction on $t$ that $R\left(K_{m+(t-1) r ! k}^{r}, A\right) \leq m+(t-1) r ! k+c(k, r)$, where $c(k, r)$ is a constant to be determined later.

Clearly this will show that $R\left(K_{n}^{r}, A\right) \leq n+c(k, r), k \mid\left(\begin{array}{l}n \\ r\end{array}\right)$. For $t=1$ we have $n=m \leq r ! k$ and $k \mid\left(\begin{array}{c}m \\ r\end{array}\right)$, clearly we may take as an initial value for $c(k, r)$ any integer larger than $R\left(K_{m}^{r}, k\right)$ $\leq R\left(K_{r ! k}^{r}, k\right)$, and in particular for $t=1$ we have $R\left(K_{m+(t-1) r ! k}^{r}, A\right) \leq m+(t-1) r ! k+c(k, r)$.

CLAIM.

$$
R\left(K_{m+t r ! k}^{r}, A\right) \leq R\left(K_{m+(t-1) r ! k}^{r}, A\right)+\left\{c(k, r)-R\left(K_{m+(t-1 r ! k}^{r}, A\right)+m+(t-1) r ! k\right\} .
$$

Indeed by induction hypothesis we conclude that $c(k, r)-R\left(K_{m+(t-1 r ! k}^{r}, A\right)+m+(t-1) r ! k \geq 0$, and although $c(k, r)$ is yet to be determined, we infer that the value of the expression of the right side of the claim is at least $R\left(K_{m+(t-1 r ! k}^{r}, A\right)$. Hence there exists a zero-sum $(\bmod A)$ copy of $K_{m+(t-1) r ! k}^{r}$ and we are left with exactly $c(k, r)$ vertices. Denote by $M$ the vertices of the zerosum copy of $K_{m+(t-1) r ! k}^{r}$ and by $N$ the remaining $c(k, r)$ vertices.

Put for $1 \leq i \leq r, E_{\mathrm{a}}=\left\{e \in E\left(K_{m+(t-1 r ! k+c(k, r)}^{r}\right):|e \cap N|=i\right\}$, that is $E_{\mathrm{t}}$ is the collection 
of all edges, in the hypergraph $K_{M \cup N}^{r}$, which contains exactly $i$ vertices in $N$ and $r-i$ vertices in $M$. Consider $E_{1}$, the set of edges with exactly one vertex in $N$. For every vertex $v \in N$ define $g_{1}(v)=\sum_{\substack{v \in e \\ e \in E_{1}}} c(e)$. Clearly if $c(k, r)$ is "large" we can find a "large" subset $N_{1} \subset N$ such that $g_{1}=g_{1}(v)=g_{1}(u)$ for every $u, v \in N_{1}$, (because $g_{1}(v) \in A,|A|=k$ ).

Consider $E_{2}$, the set of edges with exactly two vertices in $N$. For every pair of vertices $u, v \in N_{1}$ define $g_{2}(u, v)=\sum_{\substack{u, v \in \epsilon_{e} \\ e \in E_{2}}} c(e)$.

By Ramsey theorem (for pairs) if $N_{1}$ is "large" then we can find a "large" subset $N_{2} \subset N_{1}$ such that $g_{2}=g_{2}(u, v)$ is constant on $\left[N_{2}\right]^{2}$. We proceed with this argument until we reach the stage where we consider $E_{\mathrm{r}}$ apply once again the Ramsey theorem (for $r$-set) to obtain $N_{r} \subset N_{r-1} \subset N_{r-2} \subset \cdots \subset N_{1} \subset N$ such that the function $g_{\mathrm{z}}$ is constant on $\left[N_{r}\right]^{2} 1 \leq i \leq r$. So far we used only iterations of Ramsey theorem depending on $k$ - the maximum number of colors $=|A|$, and $r$.

So if $N=c(k, r)$ is sufficiently large we can have at the last stages $\left|N_{r-1}\right|=R\left(K_{r ! k}^{r}, k\right)$ and $\left|N_{r}\right|=r ! k$.

Now consider the complete $r$-uniform hypergraph with vertex set $M \cup N_{r}=Q$. Clearly $\left|M \cup N_{r}\right|=m+t r ! k$. Moreover as one can see $\sum_{e \in[Q]^{r}} c(e)=\sum_{e \in[M]^{r}} c(e)+\sum_{i=1}^{r} g_{i}\left(\begin{array}{c}r ! k \\ i\end{array}\right)$ which, by the induction step (on $[M]^{r}$ ) and lemma 1 apply to the second sum, is equal to 0 , the unit of $A$, and the claim and hence the theorem is thus proven.

REMARKS.

1. Lemma 2 is implicitly used in the form of the induction step and thus assure us that all the cases in which $k \mid\left(\begin{array}{c}n \\ r\end{array}\right)$ were considered.

2. In the process of defining $N_{r} \subset N_{r-1} \subset \cdots \subset N_{1} \subset N$ we apply $r$ times the Ramsey theorem so that the value of $c(k, r)$ obtained this way is enormous. For example for $r=2$ (i.e., graphs) we have $\left|N_{2}\right|=2 k,\left|N_{1}\right|=R\left(K_{2 k}, k\right)$, and $c(k, 2) \leq|N|$ $=k\left(\left|N_{1}\right|-1\right)+1=k R\left(K_{2 k}, k\right)-k+1$.

3. Except for the trivial fact that if $A$ is a group of order $k$ and $g \in A$ then $\underbrace{g+g+\cdots+g}_{k \text {-times }}=0$ (in $A$ ) (by Lagrange theorem). We rely in the proof only on

Ramsey theorem but not on any group theoretic concept so it will be nice to incorporate some group theoretic ideas which would result in substantially reducing the order of magnitude of $c(k, r)$.

\section{REFERENCES}

1. ÆRDÖS, P.; GINZBURG, A., \& ZIV, A., Theorem in additive number theory, Bull. Research Council Israel 10F (1961), 41-43.

2. BIALOSTOCKI, A. \& DIERKER, P., Zero sum Ramsey theorems, Congressus Numerantium 70 (1990), 193-198.

3. OLSON, J.E., On a combinatorial problem of Erdös-Ginzburg-Ziv, J. Num. Theory 8 (1976), 52-57.

4. CARO, Y., On zero-sum Ramsey numbers-complete graphs, Quart. J. Math. 43 (1992), 175181 .

5. ALON, N. \& CARO, Y., On three zero-sum Ramsey-type problems, J. Graph Theory 17 (1993), 177-192.

6. CARO, Y., Zero-sum problems - A survey, submitted.

7. BIALOSTOCKI, A.; CARO, Y. \& RODDITY, Y., On zero sum Turan numbers, ArsCombinatoria 29A (1990), 117-127 
8. BIALOSTOCKI, A. \& DIERKER, P., On zero sum Ramsey numbers: Small graphs, ArsCombinatoria 29A (1990), 193-198.

9. BIALOSTOCKI, A. \& DIERKER, P., On the Erdös-Ginzburg-Ziv theorem and the Ramsey numbers for starts and matchings, Discrete Mathematics 110 (1992), 1-8.

10. CARO, Y., On zero sum Ramsey numbers - stars, Discrete Mathematics 104 (1992), 1-6.

11. CARO, Y., On zero-sum delta systems and multiple copies of hypergraphs, J. of Graph Theory 15 (1991), 511-521. 


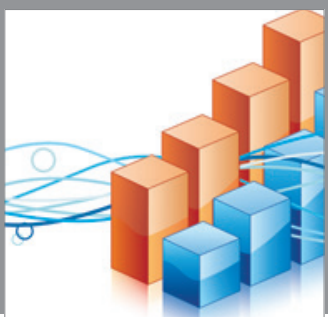

Advances in

Operations Research

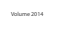

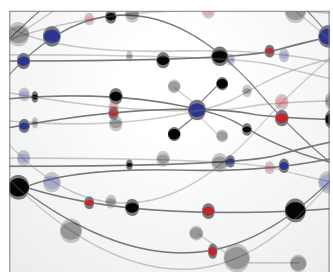

\section{The Scientific} World Journal
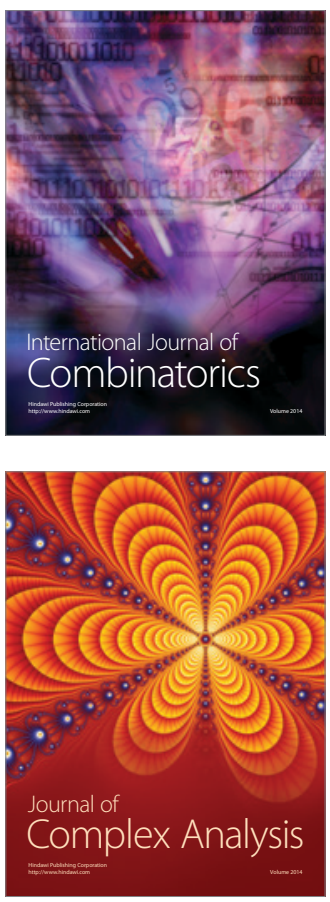

International Journal of

Mathematics and

Mathematical

Sciences
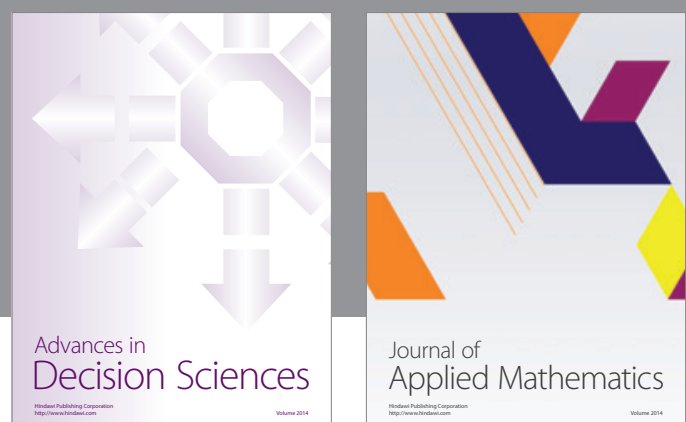

Journal of

Applied Mathematics
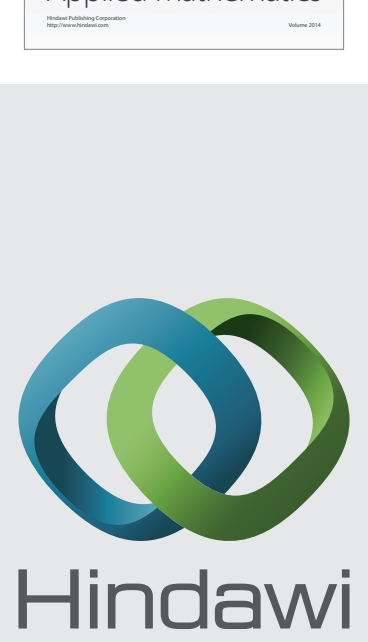

Submit your manuscripts at http://www.hindawi.com
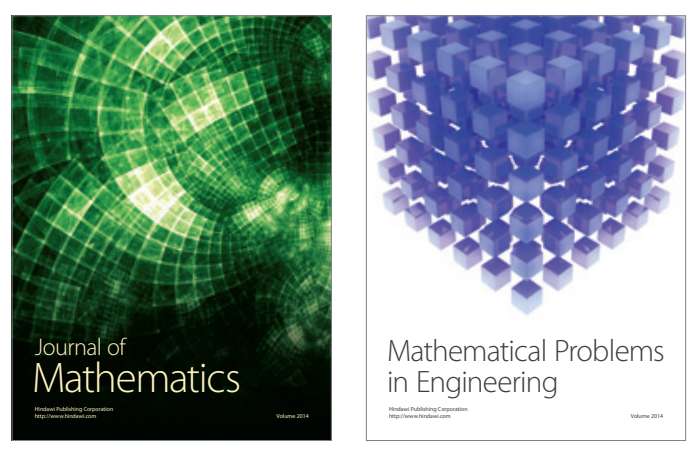

Mathematical Problems in Engineering
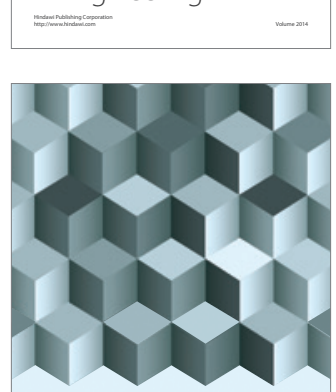

Journal of

Function Spaces
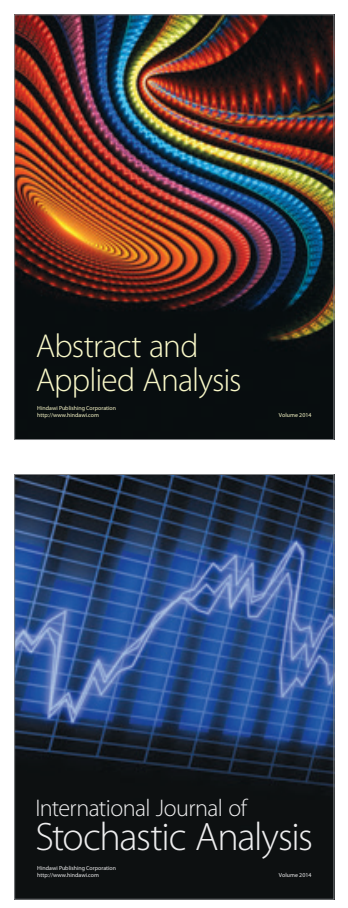

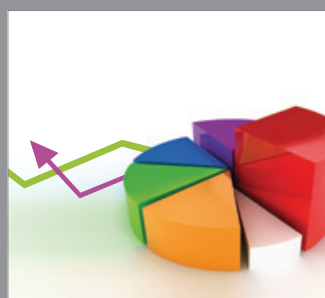

ournal of

Probability and Statistics

Promensencen
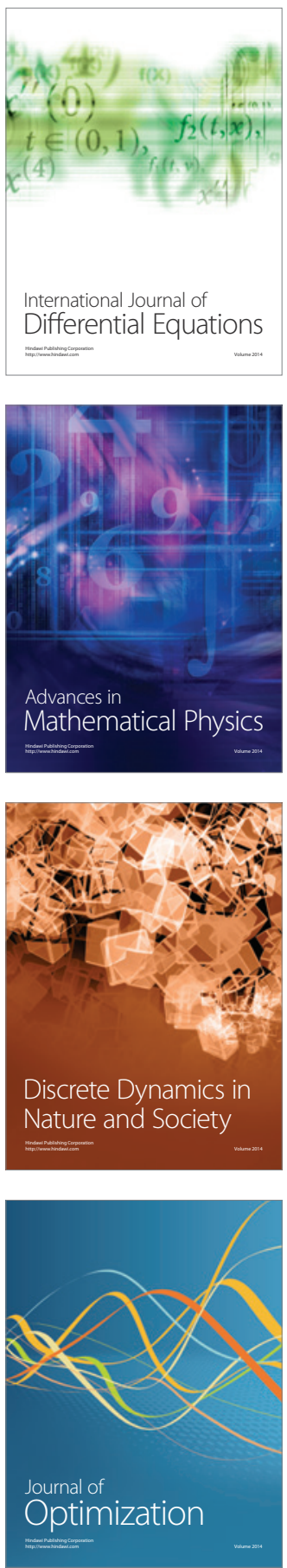